Revista de Psicología de la PUCP. Vol. XVI, 2, 1998.

\title{
SALUD MENTAL INFANTIL: TENDENCIAS ACTUALES EN LA NECESIDAD Y PROVISION DE SERVICIOS EN LOS ESTADOS UNIDOS
}

\author{
Judy Johnson Grados' \\ Universidad de John Hopkins
}

Aún cuando en los Estados Unidos existe gran demanda de servicios de salud mental infantil, tradicionalmente muchos niños no han sido atendidos adecuadamente. Esto, en parte, se debe a los obstáculos en el acceso a los servicios y al resultado de un sistema de cuidado fragmentado $y$ desorganizado. Sin embargo, en las últimas dos décadas se ha alcanzado un progreso sustancial en la manera de encarar estos problemas. Este artículo revisa la demanda de servicios de salud mental infantil que existe en los Estados Unidos y describe intentos recientes para mejorar la coordinación y el acceso al sistema de servicio de salud mental infantil. Se discuren la introducción de sistemas de cuidado, la expansión de servicios basados en la escuela y las innovaciones en la provisión de servicios a la comunidad.

Palabras clave: salud mental infantil, servicios de salud mental infantil

Children's Mental health: Current trends in the need for and provision of services in the United States traditionally many children have gone unserved. This has been partly due to barriers in accessing services and the result of a fragmented and disorganized system of care. however, in the last two decades substantial progress has been made in adressing these problems. This article reviews the need for children's mental health services in the United States, and describes recent attempts to improve coordination and access to the children's mental health service system. The introduction of systems of care, the expansion of school-based services, and innovations in the provision of community services are discussed.

Key words: children's mental health, children's mental health services

1. Recibió su doctorado en Psicología Clínica de la Universidad Estatal de Indiana en 1993. Ella trabaja como psicóloga clínica de niños y está orientada a las siguientes áreas: servicio de salud mental infantil, el afrontamiento y la adapración, y la resiliencia en niños. Actualmente es parte del equipo de la Affiliate Faculty del Departamento de Psiquiatría de la Universidad de John Hopkins en Baltimore, Maryland (EUA), y rrabaja en prácrica privada. Correo electrónico: jmgrados@welchlink.welch.jhu.edu. 

En años recientes, el aumento de las tasas de criminalidad, violencia, uso de drogas y embarazos precoces ha contribuido a la creciente crisis social de los Estados Unidos. Como resultado, muchas familias, especialmente aquellas con hijos pequeños, viven ahora bajo condiciones de severo decaimiento urbano. Los niños, vulnerables como son y que dependen para su cuidado totalmente de los adultos, poco pueden decir de sus circunstancias de vida cotidianas y están rutinariamente expuestos a tales riesgos. Por esta razón, tanto los que trabajan por el bienestar infantil como los que elaboran políticas a favor de los niños se han preocupado cada vez más por la salud y el modo de vida de los niños americanos. La coordinación y provisión de servicios de salud mental infantil constituyen importantes medidas con las que los profesionales han procurado abordar las necesidades infantiles a través del sistema de cuidado de salud.

Existe evidencia que sugiere que la necesidad de servicios de salud mental infantil se ha incrementado en las últimas dos décadas (Achenbach y Howell, 1993; Costello, 1989). Al mismo tiempo, también se ha elevado el costo de servicios de salud mental. Esto es particularrmente cierto para servicios de pacientes internos y residenciales. Como respuesta a las críricas de que los servicios institucionales de salud mental son caros y muy restrictivos, así como a la carencia de datos disponibles que documenten su eficacia (Inouye, 1988), muchos profesionales han emprendido el desarrollo de sistemas de cuidado basados en la comunidad que apoyan estancias residenciales y de pacientes internos por riempo limitado. Los servicios basados en la escuela, las innovaciones basadas en la comunidad y los servicios de prevención han concitado atención general en el campo. Este artículo revisa la demanda de servicios de salud mental infantil en los Estados Unidos así como las tendencias recientes en la provisión de servicios. 


\section{La situación de los niños americanos}

Los niños comprenden una proporción sustancial y creciente de la población de los Estados Unidos. De acuerdo al Departamento Censal de los Estados Unidos, en 1996 habían 69.4 millones de nińos menores de 18 ańos en los Estados Unidos. Para el año 2020, se estima que este número aumentará a 77.6 millones (citado en el Forum de Interagencias Federales, 1997). Aún cuando constituyen una minoría sustancial de la población, muchos niños americanos viven en familias con ingresos efectivos correspondientes al nivel de pobreza e inclusive por debajo del mismo. Además, en 1997 , el $8 \%$ de todos los niños vivía en familias con ingresos que llegaban a la mitad del nivel de pobreza, aproximadamente 7,784 dólares anuales para una familia de cuatro miembros (citado en el Forum de Interagencias Federales). Familias inestables y recursos inadecuados representan problemas significativos para la juventud actual. De acuerdo a las estadísticas de 1997, el $8 \%$ de los niños que vive en familias con bajos ingresos, a veces o frecuentemente, no tiene suficiente comida para alimentarse y el $34 \%$ de los niños vive en familias con uno o más de tres problemas de vivienda, incluyendo viviendas físicamente inadecuadas, viviendas en situación de hacinamiento o viviendas que cuestan más del $30 \%$ del ingreso familiar. Además, el porcentaje de niños menores de 18 años que vive en familias con ambos progenitores ha disminuido del $85 \%$ en 1970 a $69 \%$ en 1996 (Forum de Interagencias Federales, 1997).

No sólo los problemas económicos agobian las vidas de las familias que están criando hijos, sino que la violencia se ha convertido en un factor común. En efecro, el incremento de las tasas de violencia en los Estados Unidos ha sido considerada como una epidemia nacional (Gladwell, 1996). Los estimados de fines de la década del ochenta sugieren que las tasas de criminalidad y violencia exceden en mucho a aquéllas de otros países desarrollados de también fines de esta década. Además, este problema ha empeorado en años recientes (Fingerhut y Kleinman, 1990). Los niños son el blanco de gran parte de la violencia de los Estados Unidos de hoy. Al comparar la morralidad infantil entre ocho naciones industrializadas, los Estados Unidos se ubican en primer lugar respecto de las tasas de falle- 
cimiento asociadas a la violencia (Fingerhut y Kleinman, 1989). Un sondeo en más de 11,000 estudiantes de octavo y décimo grados encontró que el $39 \%$ de los estudiantes había tenido por lo menos una pelea física en el último año, el 33\% había recibido amenazas de daño físico, el $15 \%$ había sufrido algún robo y el $16 \%$ había sido asaltado. El colegio ofrecía escasa seguridad, el $34 \%$ de 'cs jóvenes reportó haber sido objeto de amenazas y el $13 \%$ reportó haber sido asaltado en el colegio en el año anterior (Asociación de Salud Escolar Americana, 1989). Los estudios de niños de la zona urbana presentan una imagen más deplorable todavía. De acuerdo a una investigación en estudiantes de primer y segundo grados que vivían en zonas urbanas y estaban en situación de desventaja económica, el 19\% había sido víctima de alguna forma de violencia y el $61 \%$ había sido testigo de violencia hacia otra persona (Richters y Martinez, 1993).

Los niños no solamente están expuestos a la violencia de la comunidad sino que muchos están en riesgo en su propia casa. La violencia familiar constituye una seria amenaza al bienestar emocional de los niños (Consejo de Investigación Nacional, 1993). Los estudios sugieren un número desproporcionado de niños que son testigos de violencia doméstica y que son víctimas de maltrato cada año. En 1993, los profesionales reportaron que 1.6 millones de niños fueron víctimas de abuso o negligencia. De estos niños, aproximadamente 700,000 sufrieron abuso físico, emocional o sexual (citado en el Forum de Interagencias Federales, 1997). Más perturbante todavía es el hecho que buen número de casos no son reportados, lo cual sugiere que estas cifras subestiman el número real de víctimas infantiles.

\section{La necesidad de servicios de salud mental infantil}

Dada la severidad de los estresores sociales que confrontan los nin̄os de hoy, no sorprende que los estudios comunitarios sobre la prevalencia de desórdenes psiquiátricos revelen números apreciables de jóvenes con un desorden mental diagnosticable. De acuerdo a los estimados de nueve sondeos, entre el $14 \%$ y el $22 \%$ de todos los niños sufren de desórdenes emocionales o conductuales (Anderson et al., Bird et al., 1988, Costello 
et al., 1989; Esser et al., 1990; Kashani et al., 1987; McGee et al., 1990, Offord et al., 1987). Además, se estima que cerca del 5\% de estos niños tiene un desorden mental serio, según el Manual Diagnóstico y Estadístico de Desórdenes Mentales (DSM-IV) (Asociación Psiquiátrica Americana, 1994), acompañado de incapacidad para adaptarse a ambientes familiares, escolares o comunitarios (Costello, Burns, Angold y Leaf, 1993). Más de la mitad de los niños con un desorden diagnosticable no recibe tratamiento y entre los que lo hacen, muchos reciben cuidado inapropiado (Knitzer, 1982).

Si bien pocos estudios han evaluado cambios seculares en la salud mental infantil, hallazgos preliminares de las muestras comunitarias sugieren un deterioro de la salud mental infantil con el paso del tiempo. Gould et al. (1981) reporta tasas de prevalencia de desorden psiquiátrico infantil de $12 \%$ para 1928 y 1975 . Estas tasas, según lo que se ha documentado, han sufrido un incremento de entre $14 \%$ y $20 \%$ en 1982 y 1989 y hasta de $18 \%$ a $22 \%$ de 1987 a 1989 (Costello, 1989). Achenbach y Howell informan acerca de incrementos pequeños pero penetrantes en los reportes que los padres hacen de los problemas conductuales de sus hijos así como decrementos en las competencias de sus hijos en un periodo de trece años. Estas diferencias están asociadas a síntomas particulares, como ansiedad o depresión y sugieren que no existe una sola causa que explique lo que ocurre en un determinado punto del tiempo (Achenbach y Howell, 1993). También se observan incrementos en el número de niños clasificados con síntomas en un rango clínico del desorden; en el número de niños identificados con problemas atencionales, de externalización y de internalización; y en los puntajes totales de problemas. Si bien esta evidencia indica el empeoramiento de los síntomas del niño a través del tiempo, es importante tener en mente que las variaciones en los instrumentos demográficos y de sondeo utilizados en los estudios sugieren la necesidad de cautela en las conclusiones de estos hallazgos.

También están en alza aquellas conductas de riesgo para niños y adolescentes que se sabe están asociadas a desórdenes psiquiátricos. Entre los adolescentes, sigue incrementándose las tasas de uso de drogas y alcohol 178 
(Bachman, Johnson y O'Malley, 1998; Burke, Burke, Rae y Regier, 1991). La incidencia de tasas de suicidio en los Estados Unidos ha aumentado de 4.5 por 100,000 en 1959 a 13.2 por 100,000 en 1990 en jóvenes de 15-24 ańos (Centro para el Control de Enfermedades, 1994). Asimismo, la tasa de suicidios entre varones de 15-19 años de edad ha subido de 2.6 por 100,000 en 1955 a 8.5 por 100,000 en 1987 (Brent, Perper y Allman, 1987).

\section{Sistemas de cuidado: Desarrollos en las dos últimas décadas}

Las recientes llamadas de atención sobre las necesidades de los niños con desórdenes psiquiátricos se remontan al reporte de la Comisión Conjunta de Salud Mental Infantil (1969). Este importantísimo reporte documentaba la necesidad de servicios de salud mental para niños y esbozaba los pasos necesarios para corregir el problema en los niveles federal, estatal y local. Sin embargo, no fue sino hasta 1982, con la publicación del famoso reporte de Jane Knitzer (1982) titulado Niños no reclamados que realmente comenzó el cambio en este campo. Knitzer reportó grandes brechas en las opciones de servicio disponibles para los niños así como fallas en el sistema de servicio de salud mental que no llegaba a atender las necesidades de los niños con serios desórdenes mentales. Según Knitzer, de los tres millones de niños con serios desórdenes mentales en los Estados Unidos, dos tercios no estaban recibiendo el tratamiento que requerían. Además, de los que recibían los servicios, muchos estaban recibiendo cuidado inapropiado. Knitzer consideró a estos niños como "no reclamados", abandonados por las agencias responsables de proveerles cuidado (Knitzere, 1982). Con la publicación de este importante reporte, se emprendió un movimiento para desarrollar sistemas de cuidado basados en la comunidad (Stroul y Friedman, 1986), Un año después, el Congreso promovió el desarrollo del Programa del Sistema de Servicio Infantil y Adolescente (PSSIA) a través del Instituto Nacional de Salud Mental (INSM), con fondos establecidos por primera vez en 1984.

El objetivo del Programa del Sistema de Servicio Infantil y Adolescente (PSSIA) era asistir a grupos estatales y locales en el desarrollo del 
sistema de cuidado para niños y adolescentes con serios problemas emocionales. Ahora bajo los auspicios del Centro de Servicios de Salud Mental (CSSM) `de la Administración de Servicios de Abuso de Sustancias y de Salud Mental (ASASSM), Departamento de Servicios de Salud y Humanos de los Estados Unidos, el PSSIA identificó a los niños con los problemas más serios como los de máxima prioridad. Esto estaba parcialmente de acuerdo con la filosofía que sostiene que el sector público debe servir a los niños con los problemas más severos, aparte de reconocer que el campo de la salud mental infantil requería un foco más claramente definido (Stroul y Friedman, 1986).

Específicamente, al PSSIA se le ha concedido fondos para mejorar los servicios de salud mental infantil en las siguientes formas: (a) acrecentar su liderazgo al interior de los departamentos estatales de salud mental, (b) incrementar la colaboración interagencias en las agencias de servicio infantil, (c) desarrollar la capacidad de proveer servicios de salud mental infantil basados en la comunidad, que enfaticen las opciones de tratamiento menos restrictivas y la coordinación del cuidado, (d) fortalecer el rol de las familias en el tratamiento de sus hijos y en el desarrollo del sistema de servicios infantiles, $y$ (e) hacer el sistema más responsivo a personas de variados antecedentes culturales. Una meta primaria del PSSIA era colocar cuando menos un profesional de salud mental en cada estado. Aunque originalmente los fondos federales beneficiaban 10 estados solamente, para 1993 todos los estados habían recibidos fondos del PSSIA y cada estado habia colocado por lo menos una persona de tiempo completo para que se hiciera responsable de representar los servicios de salud mental infantil (Stroul y Friedman, 1986).

Uno de los resultados del PSSIA fue la publicación en 1996 de una monografía que describía un marco de referencia para el desarrollo de un sistema de cuidado de agencias múltiples basadas en la comunidad para niños y adolescentes (Stroul y Friedman, 1986). Se define como "sistema de cuidado" al "espectro comprensivo de servicios de salud y de otros necesarios organizados en una red coordinada que satisface las múltiples y cambiantes necesidades de niños y adolescentes con severas perturbacio180 
nes emocionales y de sus familias". A menudo se confunden los términos "sistema de cuidado" y "continuo de cuidado". Un "continuo de cuidado" denota un rango de servicios mientras que "sistema de cuidado" no sólo incluye un rango de servicios sino también los componentes del programa y del servicio y la infraestructura necesaria para asegurar que los servicios se provean en una forma organizada y coherente (Stroul y Friedman, 1986). El trabajo de los autores mencionados enfatizaba la necesidad de un rango completo de servicios infantiles que trascendiera los servicios basados en la oficina y los brindados fuera del cuidado en el hogar. Los servicios recomendados incluían la incorporación de servicios de cuidado intensivo basados en el hogar, tratamiento de día, crisis y emergencia, adopción terapéutica y grupos terapéuticos. Se enfatizaba el manejo de casos como un componente crítico de la provisión de servicios y como salvaguardia para asegurar una implementación apropiada de planes de tratamiento. Además, el marco de referencia enfatizaba la necesidad de servicios comprensivos, coordinados e individualizados y propugnaba el total compromiso de las familias en el planeamiento y la ejecución de servicios. Tal marco de referencia también incorporaba mecanismos para proteger los derechos de los niños y sus familias.

Desde la implantación del PSSIA y la aparición de la monografía de Stroul y Friedman, se ha evidenciado un progreso sustancial en el desarrollo de sistemas de cuidado infantil. En la última década, se han emprendido diversos proyectos de demostración con este objetivo en mente (Attkinson, Dresser y Rosenblatt, 1991; Beachler, 1990; Behar, 1992; Cole y Poe, 1993; Bickman, 1996). Si bien una revisión cabal de estas demostraciones y evaluaciones está más allá del alcance del presente artículo, algunos proyectos merecen un breve comentario. El desarrollo del sistema de cuidado de Ventura County, California, está entre los primeros sistemas de cuidado locales. Emprendido en los 80, el modelo apuntaba a nińos en situación de máxima necesidad y tenía como metas la preservación familiar, la asistencia escolar, el rendimiento académico y prevención de la delincuencia. También intentó maximizar el funcionamiento de los niños minimizando los costos del sector público. El modelo de planeamiento 
utilizado en Ventura County maximizaba los recursos existentes reuniendo a los representantes de servicios públicos, incluyendo servicios sociales, correccionales, salud mental y educación. Después se estableció un conjunto amplio de servicios de costo-beneficio para los niños más perturbados. El modelo facilitaba considerar los múltiples problemas de los niños desde una perspectiva holística, antes que desde una perspectiva por categoría o agencia. Ventura County adoptó la filosofía de que "los niños y familias con múltiples problemas requieren soluciones interagenciales" (Stroul, Lourie, Goldman y Katz-Levy, 1992).

Si bien los fondos para tales demostraciones han provenido frecuentemente de los recursos del gobierno, algunas fundaciones privadas han contribuido también en el trabajo en esta área. En 1990, la Fundación Robert Wood Johnson, una fundación privada, tuvo la iniciativa de aportar 20 millones de dólares para desarrollar sistemas de cuidado comprensivos a través de formas novedosas de organizar y financiar los sistemas de servicio. Este proyecto fue implementado en ocho comunidades para desarrollar nuevas formas de organizar y financiar los sistemas de cuidado. El sistema de cuidado difería de acuerdo a la políitica, contexto y necesidades de cada estado (Cole y Poe, 1993). Otra fundación privada, la Fundación Annie E. Casey, ubicada en Baltimore, Maryland, otorgó fondos para una demostración que proporcionara y coordinara todas las agencias y recursos necesitados para atender a niños en desventaja económica. Finalmente, el ejército estadounidense ha emprendido una demostración en Fort Bragg, Carolina del Norte. El proyecto fue aplicado para investigar las nuevas formas de organizar y operar los sistemas de cuidado (Behar, 1992). Este modelo fue organizado basándose en el supuesto de que los nińos mejorarían si se les proporcionaba un continuo de servicios de salud mental porque así recibirían cuidado individualizado más apropiado. También se basó en el supuesto de que estos servicios serían más efectivos en sus costos que los servicios tradicionales. Sin embargo, los datos preliminares del proyecto de Fort Bragg no sugieren diferencias entre niños trat tos en el enfoque de un continuo de cuidado y niños tratados con otro enfoques (Bickman, Guthrie, Foster et al., 1995). Mientras que la 
mayoría de los proyectos de demostración se ha iniciado con fondos federales, algunos estados también ha otorgado fondos para demostraciones (hasta la fecha, las comunidades n California, Florida, Missouri, North Carolina, Ohio, Pennsylvania, Vermont y Virginia han buscado desarrollar sistemas de cuidado locales). Para una discusión cabal de estos y otros proyectos de demostración de salud mental infantil, el lector interesado puede consultar en Stroul, Lourie, Goldman y Katz-Leavy (1992) y Bickman y $\operatorname{Rog}$ (1995).

\section{Estatus actual del Sistema de Salud Mental Infantil}

Junto con la reciente reorganización del sistema de salud y la amplia incorporación de grupos de cuidado a la industria se ha puesto mayor énfasis en la efectividad de los costos y la eficiencia en la provisión de servicios de salud. Esto es cierto tanto para los servicios médicos generales así como para el sector privado. Al mismo tiempo, los temas de eficacia y contabilidad continúan copando el campo de la salud mental infantil. A mediados de la década del 80, la Oficina de Tecnología de los Estados Unidos concluyó que, "Los servicios de salud mental normalmente disponibles para los niños son inadecuados a pesar de que una base teórica e investigativa sustancial sugería que las intervenciones de salud mental infantil eran efectivas" (Oficina de Evaluación de Tecnología, 1986). Por ejemplo, en 1989 el $70 \%$ de los fondos del gobierno disponibles para los servicios de salud mental infantil fue utilizado para apoyar servicios institucionales o fuera del hogar (Burns, 1989). Al mismo tiempo, este tipo de cuidado ha sido criticado como sumamente restrictivo y demasiado usado en el servicio de las necesidades infantiles (Inouye, 1988). Además, el sistema de servicio infantil vigente (p.e. sistema de bienestar infantil, sistema educacional, sistema de cuidado de la salud y el sistema de justicia juvenil) ha sido criticado como fragmentado y desorganizado (Horwitz, Leaf, Leventhal, Forsyth y Speechly, 1992; Knitzer, 1989). 


\section{Tendencias recientes en la provisión de servicios: Mejorando el acceso al cuidado}

\section{Servicios de Salud Mental en los Colegios}

El interés en proveer servicios de salud mental desde el colegio no es nuevo. Hemos sabido por mucho tiempo que a nivel práctico, las escuelas ofrecen la ventaja de una población "cautiva" (Powell, 1991). En los Estados Unidos, a casi todos los niños y adolescentes se les exige por ley asistir al colegio. Esto significa que los niños que pueden necesitar los servicios de salud mental se encuentran en un ambiente conocido por buena parte del día, un ambiente en el que se puede acceder fácilmente a servicios in situ. Debido a que los colegios cuentan con profesionales educados en la observación del progreso y bienestar de los niños, los profesores y otros profesionales que laboran en el colegio constituyen una fuente valiosa de información respecto del avance y el estado de salud mental de los niños. En efecto, por años se ha consultado al personal del colegio para identificar los problemas de salud mental infantil (Shore y Manino, 1976).

En años recientes se ha ido perfilando el rol de los profesionales de salud mental en el ambiente escolar. Los profesionales de salud mental actualmente están pasando de un rol primario de consultor externo al rol de miembro necesario del equipo educacional del colegio. Asimismo, los servicios basados en el colegio están convirtiéndose en un lugar común en los ambientes educacionales (Dryfoos, 1994). Los servicios vinculados al colegio también están gozando de reciente popularidad. Estos servicios, a diferencia de los basados en el colegio que son proporcionados en el mismo ambiente escolar, constituyen servicios ofrecidos en otros ambientes pero con conexiones formales con el colegio. Los servicios vinculados al colegio buscan favorecer el acceso al tratamiento, reduciendo la redundancia de servicios, mejorando el manejo de casos y coordinando los recursos.

Muchas clínicas, tanto en el colegio como vinculadas al mismo, han sido organizadas como centros de tratamiento comprensivos (Defensa de 184 
la Juventud, 1994; Dryfoos, 1994). Esto porque los problemas que los jóvenes llevan a la clínica son complejos y a menudo requieren más que una intervención médica. Por ejemplo, algunas quejas físicas de los estudiantes son psicogénicas, requiriendo intervención psicológica. Además, en muchos casos, los estudiantes se presentan a las clínicas con problemas no médicos como problemas de ajuste personal, familiares y de relaciones interpersonales. En realidad, algunos estimados sugieren que hasta el 50\% de las visitas son por razones no médicas (Adelman, Barker y Nelson, 1993). Finalmente, debido a que el estigma asociado al tratamiento de salud mental no es prominente en el ambiente escolar, el colegio es el lugar ideal para la provisión de estos servicios.

El desarrollo de servicios desde el colegio, eficientes y efectivos, no carece de dificultades. Los esfuerzos por cambiar las operaciones escolares continúan encontrando resistencia. Por ejemplo, los administradores pueden creer que la incorporación de servicios psicológicos en el sistema educacional no es una parte legítima de las operaciones escolares. También, los profesores pueden considerar que el aumento en sus responsabilidades no educacionales (como proveer clínicos de salud mental y hacer referencias) aumenta igualmente su carga de trabajo ya saturada (Powell, 1991). A pesar de estos obstáculos, los colegios continúan siendo un vehículo efectivo para mejorar el acceso al cuidado y ofrecer un servicio eficiente.

\section{Manejo de Casos y Servicios "Envolventes"}

A medida que las necesidades de los niños han crecido en complejidad, se están haciendo cada vez más populares las iniciativas de salud mental basadas en la comunidad con programas ințegrales de cuidado (Clark y Clarke, 1996; Lourie, 1994; Stroul, 1993). Identificados primero por Stroul y Friedman (1986), muchos de esos modelos han empezado a funcionar a lo largo de los Estados Unidos. Estos modelos generalmente buscan coordinar todos los servicios necesarios para el niño, lo cual se hace por lo general a través de la incorporación de servicios de manejo de casos. Los que manejan casos son responsables de coordinar y organizar un equipo de profesionales, los cuales representan a cada uno de los sistemas 
con los que el niño interactúa (p.e. educacional, de salud mental, bienestar infantil, etc.). Central para el sistema de estos modelos de cuidado es el "enfoque envolvente". Esta perspectiva se refiere al proceso de abordar las necesidades de los niños a través de los dominios de vida y mediante la creación de planes de tratamiento individualizados y basados en sus necesidades (Eber y Nelson, 1997; Van DenBerg y Grealish, 1996). El enfoque envolvente involucra la creación de planes de servicio integrado únicos para los niños. Estos planes identifican las metas de los niños e incorporan tanto soportes tradicionales como no tradicionales (p.e. el uso de mentores de la comunidad, proveedores de pausas, organizaciones de padres, servicos sociales, justicia juvenil, etc.). A cada niño se le asigna un equipo de tratamiento individualizado, encabezado por el que maneja el caso, que se reúne regularmente para abordar las necesidades de tratamiento del niño. Cuando es posible, el padre es incluido en todas las reuniones de equipo y está facultado para tomar decisiones acerca del cuidado de su hijo.

Los servicios envolventes también difieren de los servicios tradicionales porque se basan en que el proceso de tratamiento debe incidir en las fortalezas del niño. La evaluación de estas fortalezas incluye la identificación de recursos individuales, familiares y de la comunidad en la vida del niño que pueden usarse como parte del plan de tratamiento. En el proceso de evaluación para identificar los recursos del niño, se exploran la historia, estructura, relaciones y funcionamiento de la familia. Esto difiere sustancialmente del plan de tratamiento tradicional, en el que los problemas constituyen el único foco de la intervención.

\section{Intervención Móvil de las Crisis}

Otra reciente innovación en el sistema de servicio comunitario ha sido la introducción de servicios móviles para las crisis. Tales servicios han sido emprendidos con el objetivo de incrementar el acceso a los servicios. Éstos son servicios de respuesta a emergencias que funcionan las 24 horas del día y están diseñados para proveer intervención en situaciones de crisis que se dan en la comunidad. Asimismo, cuentan con equipos que incluyen 186 
psicólogos, psiquiatras, enfermeras registradas, consejeros y trabajadores sociales. Normalmente, luego de la referencia hecha por algún miembro preocupado de la familia, vecino o agencia social, el personal entrenado proporciona la intervención que tiene a su alcance, entrevistándose con el niño/ familia en su hogar o en otro lugar de la comunidad.

La intervención móvil de las crisis ofrece algunas ventajas respecto del servicio de salud mental tradicional. Primero, muchas familias en crisis son incapaces de navegar en las complejidades que implican enrolarse en el sistema de salud mental (esto es, obtener fondos para el seguro médico, coordinar transporte, etc.). La intervención móvil de las crisis hace posible que los servicios estén disponibles para aquellas familias que necesitan asistencia inmediata y que de otro modo, no recibirían cuidado. Segundo, debido a que son proporcionadas sobre la base de una emergencia, los servicios móviles para las crisis se ofrecen de una manera expeditiva. Esto contrasta con las muy conocidas listas de espera hasta por meses enteros que confrontan familias que buscan servicios externos tradicionales. Las familias que se muestran ambivalentes acerca de enrolar a su hijo en servicios organizados pueden presentar una mejor disposición para hacerlo si son atendidas inmediatamente durante una crisis, que si tienen que esperar un periodo largo antes de ser atendidas. Tercero, la visita domiciliaria por parte de un profesional puede ser mucho menos perturbante y desorganizante para el niño y su familia que la alternativa de esperar por horas en un ocupado departamento/clínica de emergencia. Finalmente, los servicios móviles de las crisis benefician no sólo a los consumidores sino también ayudan a mejorar los servicios ofrecidos por el profesional. Las visitas domiciliarias durante el proceso diagnóstico proveen al profesional información adicional con la cual puede hacer una evaluación más precisa del niño y de su ambiente familiar (Zealberg, 1993).

\section{El Rol de la Prevención en los Servicios de Salud Mental}

Las iniciativas de prevención se han dividido tradicionalmente en tres niveles: Prevención primaria, secundaria y terciaria. Sin embargo, diferen- 
ciar estos servicios ha sido a veces una tarea difícil. La "prevención primaria" ha sido usada para referirse a aquellos servicios que abordan las causas del desorden. Según Caplan (1961), la prevención primaria es “...la promoción de la salud y la protección contra la posibilidad de desorden mental originado por medidas que interfieren el equilibrio del organismo y del ambiente antes del inicio de la condición patológica". Causas previsibles de desorden pueden oscilar desde deficiencias nutricionales hasta la ausencia de la crianza materna requerida para un desarrollo humano saludable. La "prevención secundaria" está fundamentalmente ocupada en la detección temprana de la enfermedad. Tal intervención ocurre antes del inicio de los síntomas serios. Finalmente, la "prevención terciaria" se refiere a servicios de salud mental tradicionales, a la rehabilitación de individuos de acuerdo a los diagnósticos del desorden.

La analogía de un río (tal como la presentó Simeonson, 1994) puede ser utilizada para ilustrar las diferencias entre estos servicios/intervenciones. Primero, río abajo podemos encontrar muchos jóvenes "ahogándose", son aquellos niños que necesitan de los servicios y que han sucumbido a problemas emocionales o conductuales (prevención terciaria). Río arriba puede haber menos actividad y la gente puede estar parada a la orilla del río tratando de determinar si los niños están nadando o están "en riesgo" de ahogarse y necesitan rescate. Este punto en el río representa el punto de intervención temprana e incluye programas que buscan identificar los desórdenes, los retardos evolutivos y los problemas de aprendizaje (prevención secundaria). Más río arriba, los niños pueden estar saltando o cayéndose al río por alguna combinación de disfunción familiar o de la comunidad. Estos niños representan a las jóvenes embarazadas con un cuidado prenatal pobre, a jóvenes que van a ser padres por primera vez y que no saben casi nada sobre sus funciones, etc. (prevención primaria). Poco se hace para enseńarles "a nadar" o a evitar que se "caigan" al río.

Los programas de prevención se han implementado ampliamente en los Estados Unidos. Los sondeos de los currícula de colegios públicos y privados indican que casi todos los colegios elementales, intermedios y superiores ofrecen un número de estos programas. Frecuentemente los 
tópicos cubiertos incluyen prevención de drogas y alcohol, uso de tabaco, prevención del Sida, dieta y nutrición, salud mental y emocional, prevención del embarazo, prevención de la violencia y prevención de daños (Collins et al., 1995). Los programas de prevención comunitaria poseen también una amplia oferta. Los padres participan en cerca de 3,000 programas de prevención de drogas basados en la comunidad (Klitzner, Gruenwald y Bamberger, 1990) y cerca de dos tercios de todas las compañias patrocinan más de 50 programas de salud orientados a la prevención infantil (Simmons, 1993).

El siguiente paso en el mejoramiento de los servicios de salud mental infantil puede ser el desarrollo de planes de tratamiento integrados, que no sólo incorporen sistemas de servicio múltiple, sino que también utilicen intervenciones primarias, secundarias y terciarias en el tratamiento de los problemas infantiles. Hasta hace poco, los servicios de salud mental solamente tenían como blancos los síntomas de desorden mental. Sin embargo, asumir que es posible prevenir el desorden se revela como fundamental para comprender el tratamiento de problemas emocionales y conductuales. La integración de tales servicios permitiría desarrollar planes de tratamiento que tuvieran como meta, tanto los problemas conductuales o emocionales inmediatos de los niños, como otros factores de riesgo que se sabe contribuyen al desorden mental (p.e. poco control por parte de los padres, nutrición deficiente, necesidades educacionales respecto del alcohol y las drogas). Por ejemplo, un niño que necesita servicios por un Desorden de Déficir Atencional puede beneficiarse asimismo de una intervención preventiva que apunte hacia los factores de riesgo únicos al niño. Las intervenciones pueden incluir educación en drogas/alcohol, programas de prevención de la violencia, etc. Tal estrategia no sólo podría beneficiar la salud y el desarrollo de los niños sino que también podría demostrar ser efectiva en cuanto costos en la larga carrera de la prevención del inicio de un desorden y la determinación de la necesidad futura de servicios. 


\section{Conclusión}

En suma, la necesidad de servicios mentales infantiles se ha incrementado en los últimos años y en las dos últimas décadas el campo ha crecido bastante. Con todo, queda mucho por hacer. El desarrollo e implementación de proyectos de demostración de servicios a lo largo del país han servido como un primer paso importante en la facilitación del desarrollo de este campo. Ahora, se requiere una rigurosa evaluación de tales proyectos. Con pocas excepciones, los proyectos de demostración han considerado la prestación de servicios y el desarrollo de programas como prioridades respecto de las actividades de evaluación. La evaluación de tales proyectos requerirá que los investigadores aborden los temas de definición y medición de constructos complejos, como el proceso de prestación de servicios, la eficacia de la psicoterapia y los logros infantiles. Esta es una tarea atemorizante que requiere de amplios recursos fiscales, así como grandes cantidades de esfuerzo por parte de los que están trabajando en el campo. Además, las innovaciones en los servicios habituales, como los servicios desde el colegio, el manejo de casos y la intervención móvil de las crisis, han preparado el camino para los cambios en las formas de pensar acerca de la prestación de servicios de salud mental infantil. El desarrollo continuo de estos y otros enfoques de costo -efectivo acercará a este campo un paso más al tratamiento adecuado de las necesidades de salud mental de los niños en los Estados Unidos.

\section{Referencias}

Achenbach, T. M. y Howell, C. T. (1993). Are American children's problems getting worse? A 13-year comparison. Journal of the American Academy of Child \& Adolescent Psychiatry, 32(6), 1145-54.

Adelman, H. S., Barker, L. A. y Nelson, P. (1993). A study of a schoolbased clinic: Who uses it and who doesn't? Journal of Clinical Child Psychology, 22, 52-59. 
Advocates for Youth (1994). School-based and school-linked health centers: The facts. Washington, DC: Autor.

American Psychiatric Association (1994). Diagnostic and Statistical Manual of Mental Disorders (4 ed). Washington, DC: American Psychiatric Press.

American School Health Association (1989). Oakland, CA: Third Party. Anderson, J. C., Williams, S., McGee, R. y Silva, P. A. (1987). DSMIII disorders in preadolescent children: Prevalence in a large sample from the general population. Archives of General Psychiatry, 44(1), 6976.

Attkinson, C., Dresser, K. y Rosenblatt, A. (1991). Service systems for youth with severe emotional disorder: Systems of care research in California. En U.S. House of Representatives, Close to home: Community-based Mental Health Services for Children, Hearing Before the Select Committee on Children, Youth, and Families, U.S. House of Representatives (pp. pp. 96-130). Washington, DC: Government Printing Office.

Bachman, J. G., Johnson, L. D. y O’Malley, P. M. (1998). Explaining recent increases in students' marijuana use: Impact of perceived risks and disapproval, 1976 through 1996. American Journal of Public Health, 88(6), 887-92.

Beachler, M. (1990). The mental health services program for youth. Journal of Mental Health Administration, 17, 115-121.

Behar, L. (1992). Fort Bragg Child and Adolescent Mental Health Demonstration. Raleigh: North Carolina Division of Mental Health, Developmental Disabilities, and Substance Abuse Services, Child and Family Services Branch.

Bickman, L. (1996). A continuum of care: More is not always better. American Psychologist, 51(7), 689-701.

Bickman, L., Guthrie, P. y Foster, E. (1995). Managed care in mental health: The Fort Bragg experiment. Nueva York: Plenum Press.

Bickman, L. y Rog, D. (1995). Children's mental health services: Research, policy, and evaluation. Thousand Oaks, CA: Sage publications. 
Bird, H. R., Canino, G., Rubio-Stipec, M., Gould, M., Ribert, J., Sesman, M., Woodbury, M., Huertas-Goldman, S., Pagan, A., Sanches-Lacay, A. y Moscoso, M. (1988). Estimates of the prevalence of childhood maladjustment in a community survey in Puerto Rico. Archives of General Psychiatry, 45, 1120-1126.

Brent, D. A., Perper, J. A. y Allman, C. J. (1987). Alcohol, firearms, and suicide among youth. Temporal trends in Allegheny County, Pennsylvania, 1960 to 1983. JAMA, 257(24), 3369-72.

Burke, K. C., Burke, J. D. Jr, Rae, D. S. y Regier, D. A. (1991). Comparing age at onset of major depression and other psychiatric disorders by birth cohorts in five US community populations. Archives of General Psychiatry, 48(9), 789-95.

Burns, B. J. (1989). Critical research directions for child mental health services. En Second Annual Conference Proceedings (pp. 8-13). Florida Mental Health Institute.

Caplan, G. (1964). The principles of preventive psychiatry. Nueva York: Basic Books.

Caplan, G. (1961). Prevention of mental disorders in children. Nueva York: Basic Books.

Centers for Disease Control (1994). Programs for the prevention of suicide among adolescents and young adults. Morbidity and Mortality Weekly Reports, 43(SS-1), 1-7.

Clarke, H. B. y Clarke, R. T. (1996). Research on the wraparound process and individualized services for children with multi-system needs. Journal of Child and Family Studies, 5, 1-5.

Cole, R. y Poe, S. (1993). Partnerships for care: Systems of care for children with serious emotional disturbances and their families. Washington, DC: Washington Business Group on Health, Mental Health Services Program for Youth.

Collins, J. L., Small, M. L., Kann, L., Pateman, B. C., Gold, R. S. y Kolbe, L. J. (1995). School Health Education. Journal of School Health, 65, 302-311.

Costello, E. J. (1989). Developments in child psychiatric epidemiology. Journal of the American Academy of Child and Adolescent Psychiatry, 28(6), 836-41. 
Costello, E. J., Burns, B. J., Angold, A. y Leaf, P. J. (1993). How can epidemiology improve mental health services for children and adolescents? Journal of the American Academy of Child \& Adolescent Psychiatry, 32(6), 1106-14; discussion 1114-7.

Dryfoos, J. (1994). Full-service schools: A revolution in health and social services for children, youth, and families. San Francisco: Jossey-Bass. Eber, L. y Nelson, C. (1997). School-based wraparound planning: Integrated services for students with emotional and behavioral needs. American Journal of Orthopsychiatry, 67(3), 385-395.

Esser, G., Schmidt, M. H. y Woerner, W. (1990). Epidemiology and course of psychiatric disorders in school-age children-results of a longitudinal study. Journal of Child Psychology and Psychiatry and Allied Disciplines, 31(2), 243-63.

Federal Interagency Forum on Child and Family Statistics (1997). Washington, DC: Federal Interagency Forum on Child and Family Statistics.

Fingerhut, L. A. y Kleinman, J. C. (1990). International and interstate comparisons of homicide among young males. Journal of the American Medical Association, 263(24), 3292-5.

Fingerhut, L. A. y Kleinman, J. C. (1989). Trends and current status in childhood mortality, United States, 1900-1985. En Vital Health Statistics (Vol. 3, Chap. 26, ). Washington DC: National Center for Health Statistics.

Gladwell, M. (1996). The tipping point. The New Yorker 2 32-38.

Gould, M. S., Wunsch-Hitzig, R. y Dohrenwend, B. (1981). Estimating the prevalence of childhood psychopathology: A critical review. Journal of the American Academy of Child Psychiatry, 20(3); 462-76.

Horwitz, S. M., Leaf, P. J., Leventhal, J. M., Forsyth, B. y Speechley, K. N. (1992). Identification and management of psychosocial and developmental problems in community-based, primary care pediatric practices. Pediatrics, $89(3), 480-5$.

Inouye, D. K. (1988). Children's mental health issues. American Psychologist, 43(10), 813-6. 
Joint Commission on the Mental Health of Children (1969). Crisis in child mental health. Nueva York: Harper and Row.

Kashani, J., Beck, N., Hoeper, E., Fallahi, C., Corcoran, C., McAllister, J., Rosenberg, T. y Reid, J. (1987). Psychiatric disorders in a community sample of adolescents. American Journal of Psychiatry, 144(5), 584-589.

Klitzner, M., Gruenewald, P. J. y Bamburger (1990). The assessment of parent-led prevention programs: A national descriptive study. Journal of Drug Education, 20(2), 111-25.

Knitzer, J. (1989). Children's mental health: the advocacy challenge. En R.M. Friedman, A.J. Duchnowski y E.L. Henderson (Eds.), Advocacy on behalf of children with serious emotional problems. Springfield, Illinois: Charles C. Thomas.

Knitzer, J. (1982). Unclaimed children: The failure of public responsibility to children and adolescents in need of mental health services. Washington, DC: Children's Defense Fund.

Lourie, I. (1994). Principles of local system development for children, adolescents, and their families. Chicago, Illinois: Kaleidoscope.

McGee, R., Feehan, M., Williams, S., Partridge, F., Silva, P. A. y Kelly, J. (1990). DSM-III disorders in a large sample of adolescents. Journal of the American Academy of Child \& Adolescent Psychiatry, 29(4), 6119.

National Research Council, Panel on Research on Child Abuse and Neglect (1993). Understanding child abuse and neglect. Washington, DC: National Academy Press.

Office of Technology Assessment (1986). Children's mental health: Problems and services. Washington, DC: Autor.

Offord, D. R., Boyle, M. H., Szatmari, P., Rae-Grant, N. I., Links, P. S., Cadman, D. T., Byles, J. A., Crawford, J. W., Blum, H. M., Byrne, C., et al. (1987). Ontario Child Health Study. II. Six-month prevalence of disorder and rates of service utilization. Archives of General Psychiatry, 44(9), 832-6.

Powell, D. R. (1991). How schools support families: Critical policy tension. Elementary School Journal, 91, 307-319. 
Richters, J. E. y Martinez, P. (1993). The NIMH community violence project: $I$. Children as victims of and witnesses to violence. Psychiatry, 56(1), 7-21.

Shore, M. F. y Marino, F. V. (1976). Mental health services for children and youth. Journal of Clinical Child Psychology, 5, 21-25.

Simeonsson, R. J. (1994). Risk, resilience, \& prevention: Promoting the wellbeing of all children. Baltimore, MD: Paul H. Brooks Publishing.

Simmons, S. J. (1993). The economics of prevention. En R.N. Knollmueller 26(Ed.), Prevention across the lifespan: Healthy people for the twentyfirst century (pp. 1-9). Washington, DC: American Nurses Publishing. Stroul, B. A. (1993). A system of care for children and adolescents with severe emotional disturbances: What are the results? Washington, DC: CASSP Technical Assistance Center/Center for the Child Health and Mental Health Policy/Georgetown University Child Development Center.

Stroul, B. A. y Friedman, R. M. (1986). A system of care for severly emotionally disturbed children and youth. Washington, DC: Georgetown University, Child and Adolescent Service System Program Technical Assistance Center.

Stroul, B., Lourie, I., Goldman, S. y Katz-Leavy, J. (National Technical Assistance Center for Children's Mental Health). Profiles of local systems of care for children and adolescents with severe emotional disurbances. Washington, DC: Georgetown University Child Development Center.

VanDenBerg, J. E. y Grealish, E. M. (1996). Individualized services and supports through the wraparound process: Philosophy and procedures. Journal of Child and Family Studies, 5(1), 7-21.

Zealberg, J. J., Santos, A. B. y Fisher, R. K. (1993). Benefits of mobile crisis programs. Hospital and Community Psychiatry, 44(1), 16-17. 